\title{
Efficiency improvement and spectral shift of an organic light-emitting device with a square-based microlens array
}

\author{
Mao-Kuo Wei ${ }^{\mathrm{a}, *}$, Hoang-Yan Lin ${ }^{\mathrm{b}}$, Jiun-Haw Lee ${ }^{\mathrm{b}}$, Kuan-Yu Chen ${ }^{\mathrm{b}}$, Yu-Hsuan Ho ${ }^{\mathrm{b}}$, \\ Ciao-Ci Lin ${ }^{a}$, Chia-Fang $\mathrm{Wu}^{\mathrm{b}}$, Hung-Yi Lin ${ }^{\mathrm{c}}$, Jen-Hui Tsai ${ }^{\mathrm{c}}$, Tung-Chuan $\mathrm{Wu}^{\mathrm{c}}$ \\ a Institute of Opto-Electronic Engineering, Department of Materials Science and Engineering, National Dong Hwa University, Hualien 974, Taiwan, ROC \\ ${ }^{\mathrm{b}}$ Graduate Institute of Photonics and Optoelectronics, Department of Electrical Engineering, National Taiwan University, Taipei 106, Taiwan, ROC \\ ${ }^{c}$ Mechanical and Systems Research Laboratories, Industrial Technology Research Institute, Hsinchu 310, Taiwan, ROC
}

\section{A R T I C L E I N F O}

Article history:

Received 18 September 2007

Received in revised form 22 July 2008

Accepted 25 July 2008

\section{PACS:}

42.79.Bh

85.60.Jb

$85.85 .+\mathrm{j}$

Keywords:

Microlens

Organic light-emitting device

Luminous current efficiency

Luminous power efficiency

Fill factor

Height ratio

\begin{abstract}
A B S T R A C T
In this paper, we present and analyze the influences of the fill factor and the height ratio of square-based microlens arrays on the optical characteristics of an organic light-emitting device (OLED). These properties include spectral shift, CIE coordinates, viewing-angle dependence, luminous current efficiency and luminous power efficiency. Both the luminous current efficiency and luminous power efficiency of the OLED were found to increase linearly with increasing the fill factor of microlenses. It is also found that the full width at half maximum (FWHM) of the OLED spectra and CIE coordinate decreased linearly with increasing the fill factor of the microlenses. Besides, the efficiency improvement of the OLED increased with the height ratio of attached microlenses. Compared to the OLED, the luminous current efficiency and luminous power efficiency of the device can be enhanced by $42 \%$ and $47 \%$, respectively, by attaching the microlens array having a fill factor of $90 \%$ and a height ratio of 0.57 . We also observed blue shifts at different viewing angles when microlens arrays were attached to the OLED, which is the evidence that the waveguiding modes are being extracted. In our planar OLED, the peak wavelength blue shifted and the FWHM decreased with increasing the viewing angles due to the microcavity effect.
\end{abstract}

() 2008 Elsevier B.V. All rights reserved.

\section{Introduction}

The organic light-emitting devices (OLEDs) are planar light sources with medium luminance in nature. They are energy-saving, thin, light-weight and environmentally friendly. The most important advantage of the OLEDs is that they do not require any backlight modules, the thickness of the OLEDs could thus be much less than that of liquid crystal displays (LCDs). Therefore, the OLEDs are more and more popularly applied in the displays for mobile electronics.

Since only approximate $20 \%$ of the internally emitted light can be released to outside environment [1], many techniques based on destroying substrate waveguiding phenomena have been studied [2-15], in order to improve the efficiency of the OLEDs. Among these techniques, microlens arrays [8-13] seem to be a good choice due to their simple and reliable manufacturing process. For the

\footnotetext{
* Corresponding author. Tel.: +8863 8634221; fax: +88638634200.

E-mail address: mkwei@mail.ndhu.edu.tw (M.-K. Wei).
}

attachment of microlens array, it showed that the luminous current efficiency of the OLED increased linearly with increasing the fill factor of microlenses [9,12,13]. Greiner also used a Monte Carlo raytracing simulation method to show that the efficiency of the OLED increased with increasing both the fill factor and the height ratio of microlenses, and also increased with increasing the reflectance of the metallic cathode of the OLED [14].

The three frequently used base shapes of microlenses are circle, hexagon and square. Before thermal reflow process, photoresist plates with base shapes of hexagon and square can have higher fill factors than those with a base shape of circle. Technologically, these plates with a hexagonal base can be economically made only by photolithography. The size of these hexagon-based plates is thus limited to the capability of aligners and steppers. On the other hand, the photoresist plates with a square base can be produced not only by photolithography but also by laser micromachining and exposure. The size of these square-based plates can be unlimited. This means that the large-sized, square-based microlens array can be possibly manufactured. For this reason, the square-based microlens arrays will be focused on in this study. 
In this work, squared-based microlens arrays were made by the combination of photolithography, thermal reflow, molding and UV forming techniques. They were used to improve the luminous current efficiency normal to the device surface and the luminous power efficiency in purpose. The parameters of microlens arrays include the fill factor, edge length of the base region, and lens sag. Additionally, the influences of the microlens parameters on the optical spectrum and CIE coordinate of the OLED have also been systematically studied. Since outcoupling efficiency enhancement depends on the angular intensity and spectral distribution of the OLED, the device structure of this study was fixed the same as our previous work [15] for a quantitative comparison.

\section{Background}

If a rectangular photoresist plate has a length, width and thickness of $l_{\mathrm{PR}}, w_{\mathrm{PR}}$ and $d_{\mathrm{PR}}$, respectively, to be heat treated, a cylindrical lens having a sag and a radius of curvature of $h_{\text {lens }}$ and $r_{\text {lens, }}$, respectively, will be formed after fully thermal reflow. If there is no edge broadening and no volume shrinkage after thermal reflow, and $l_{\mathrm{PR}}>>w_{\mathrm{PR}}$, the relationships among $r_{\text {lens }}, h_{\text {lens }}, d_{\mathrm{PR}}$ and $w_{\mathrm{PR}}$ can then be obtained through simply geometrical consideration $[16,17]$

$r_{\text {lens }}=\frac{w_{\text {PR }}^{2}}{8 h_{\text {lens }}}+\frac{h_{\text {lens }}}{2}$

$d_{\mathrm{PR}}=\frac{r_{\text {lens }}^{2}}{w_{\mathrm{PR}}} \sin ^{-1}\left(\frac{w_{\mathrm{PR}}}{2 r_{\text {lens }}}\right)-\frac{1}{2}\left(r_{\text {lens }}-d_{\text {lens }}\right)$

If the driving current and the active area of the OLED are kept the same, the luminance ratio of the device with microlens array $\left(L_{\text {lens }}\right)$ to that without microlenses $\left(L_{0}\right)$ should equal the ratio of their luminous current efficiencies [15],

$\frac{\eta_{L, \text { lens }}}{\eta_{L, 0}}=\frac{L_{\text {lens }}}{L_{0}}$

where $\eta_{L \text {,lens }}$ and $\eta_{L, 0}$ are the luminous current efficiencies of the devices with and without microlenses, respectively.

If the driving current, voltage and the active area of the OLED device are kept unchanged, the ratio of the luminous power efficiencies of the device with microlens array $\left(\eta_{\mathrm{P}, \text { lens }}\right)$ to one without microlenses $\left(\eta_{\mathrm{P}, 0}\right)$ should be equal to the ratio of their luminous exitances [15]

$\frac{\eta_{\mathrm{P}, \text { lens }}}{\eta_{\mathrm{P}, 0}}=\frac{\int_{0^{\circ}}^{90^{\circ}} L_{\text {lens }}(\theta) \sin \theta \mathrm{d} \theta}{\int_{0^{\circ}}^{90^{\circ}} L_{0}(\theta) \sin \theta \mathrm{d} \theta}$

where $L_{\text {lens }}(\theta)$ and $L_{0}(\theta)$ are the angular-dependent luminance of the device with and without microlens array, respectively.

\section{Experimental methods}

The process flow for making free-standing films with microlens array was shown in Fig. 1. First, a (100) silicon wafer was used as a substrate. Second, the photoresist AZ P4620 was spun on the wafer and through photolithographic process to make square plates with a thickness of $8.9 \mu \mathrm{m}$. Third, these plates were thermally reflowed to have a spherical cap contour. Fourth, liquid polydimethylsiloxane (PDMS) mixed with its hardener were poured on the wafer and put in an oven to be thermally cured. Fifth, the PDMS mold was released by peeling off the wafer and the mold. Sixth, UV-curable epoxy OG152 (Epoxy Technology, Inc.) was coated on the PDMS mold and then exposed to UV radiation to harden the OG152. According to the data sheet, the OG152 has a refractive index of 1.5303 and a transmittance of $\sim 100 \%$ in the wavelength range of $450-900 \mathrm{~nm}$. Finally, a free-standing epoxy film with the duplicated microlens array on the film surface was formed after separating the mold from the film.
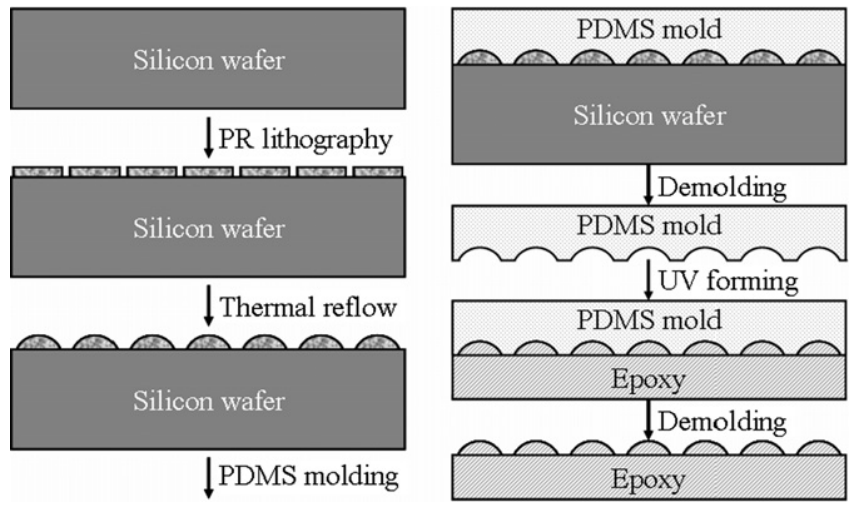

Fig. 1. Schematics of the process flow for microlens-array duplication.

In this study, two sets (RO35.4 and RI50) of microlens arrays were made, which possessed edge lengths of 35.4 and $50.0 \mu \mathrm{m}$, respectively. The microlenses were arranged in square arrays. The gap distance between two adjacent microlenses was kept constant in each set of microlens arrays. Since the edge length and thickness of photoresist plates in each set of microlens arrays remained constant, the geometrical contours should be the same after thermally reflowing these plates. The fill factor $(F)$, defined as the ratio of the total areas of the base region of microlenses to that of the device's emitting surface, was adjusted in the range between $20 \%$ and $90 \%$ by varying the gap distance in each set of microlens arrays. The parameters of theses microlens arrays were illustrated in Table 1.

The device structure of the green OLED was ITO $(110 \mathrm{~nm}) / \mathrm{TNB}$ $(80 \mathrm{~nm}) / \operatorname{Bebq} 2(80 \mathrm{~nm}) / \mathrm{LiF}(0.7 \mathrm{~nm}) / \mathrm{Al}(100 \mathrm{~nm})$, as described in [15]. The thickness of the glass substrate was $0.7 \mathrm{~mm}$ and the active area of the OLED device was $10 \times 10 \mathrm{~mm}^{2}$. The luminance of the OLED device without any microlenses was first measured as a reference. To reduce the influence of ambient light on the CIE coordinate, the luminance of the OLED device without microlenses was set at $\sim 680 \mathrm{~cd} / \mathrm{m}^{2}$. The silicone oil with a refractive index of $\sim 1.5$ was then used to reduce the reflection between the duplicated film and the glass substrate prior to the optical characterizations. All the microlens arrays were attached in turn on the same OLED device to minimize measuring errors from different OLED panels.

A source meter (Keithley 2400) was used to supply the driving current for the OLED device and to monitor both the current and voltage of the device at the same time. A spectroradiometer (Minolta CS-1000A) was focused on the active area of the device to measure its luminance, optical emitting spectrum and CIE coordinate. The resolution of the measured spectrum was set at $1 \mathrm{~nm}$. For the angular-dependent luminance measurement, the device was fixed on a rotational stage, and the luminance was then measured at different viewing angles from $0^{\circ}$ to $85^{\circ}$.

The edge lengths and gap distances of the photoresist plates were measured using a surface profiler (Tencor, Alpha-Step 500). The sag of microlenses was measured with a scanning electron

Table 1

The parameters of the two sets of microlens arrays

\begin{tabular}{lll}
\hline Set no. & RO35.4 & RI50 \\
\hline Thickness of photoresist plates, $d_{\mathrm{PR}}(\mu \mathrm{m})$ & 8.9 & 8.9 \\
Edge length of photoresist plates, $l_{\mathrm{PR}}(\mu \mathrm{m})$ & 35.4 & 50 \\
Gap distance, $G(\mu \mathrm{m})$ & $2.0-43.7$ & $2.7-61.7$ \\
Height of microlenses, $h_{\text {lens }}(\mu \mathrm{m})$ & $12.9 \pm 0.2$ & $13.6 \pm 0.2$ \\
Radius of curvature of microlenses, $r_{\text {lens }}(\mu \mathrm{m})$ & $22.5 \pm 0.3$ & $31.2 \pm 0.2$ \\
Height ratio of microlenses, $h_{\text {lens }} / r_{\text {lens }}$ & $0.57 \pm 0.01$ & $0.44 \pm 0.01$ \\
\hline
\end{tabular}


microscope (SEM, Hitachi S3500). The radius of curvature of microlenses was analyzed by curve fitting the microlens contour obtained from the SEM microphotograph.

\section{Results and discussion}

The fill factor $(F)$ of a microlens array can be derived from a simple geometrical relationship

$F=\left(\frac{l_{\mathrm{PR}}}{l_{\mathrm{PR}}+G}\right)^{2}$

where $l_{\mathrm{PR}}$ and $G$ are the edge length and the gap distance, respectively. As $G$ approaches zero, $F$ approaches unity. In this condition, the duplicated epoxy film is fully covered with microlenses.

It is difficult to analyze the exact surface contour of a squarebased microlens, but may roughly estimate the sag and the radius of curvature through simulation of a cylindrical microlens. Supposed that the length and width are equal, and the thickness of photoresist plate is $8.9 \mu \mathrm{m}$, the variations of the sag, the radius of curvature and the height ratio with the edge length can be calculated from Eqs. (1a) and (1b), as shown in Fig. 2. It showed that both the sag and the radius of curvature of microlenses increased with increasing the edge length of the photoresist plates. The height ratio of microlenses, however, decreased with increasing the edge length of these plates.

Typical duplicated free-standing epoxy films with square-based microlens arrays, having a fill factor of $60 \%$, on their surface were shown in Fig. 3. These microlenses revealed excellent spherical contours. In this study, the radius of curvature and the sag were analyzed by curve fitting the microlens contour obtained from SEM microphotograph. Actually, they were calculated by averaging

a
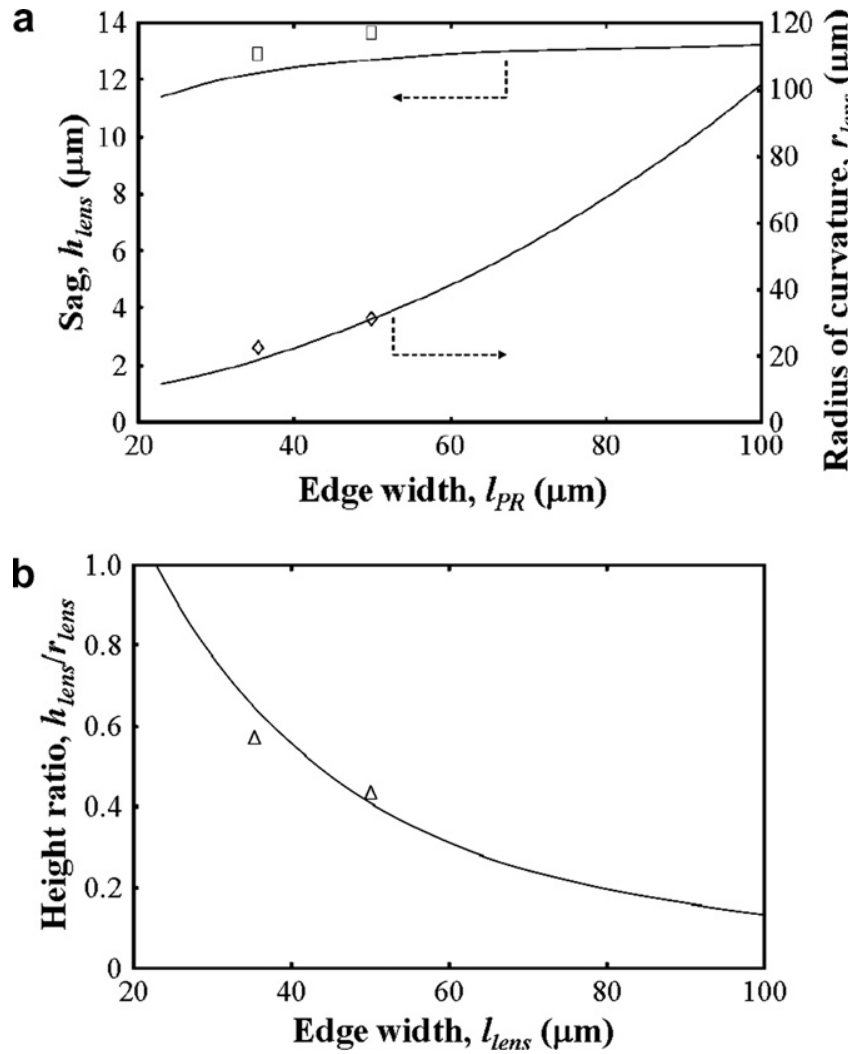

Fig. 2. The variations of (a) the sag and the radius of curvature and (b) the height ratio of microlenses with the edge length of photoresist plates. The lines and symbols represent simulated and experimental results, respectively. a

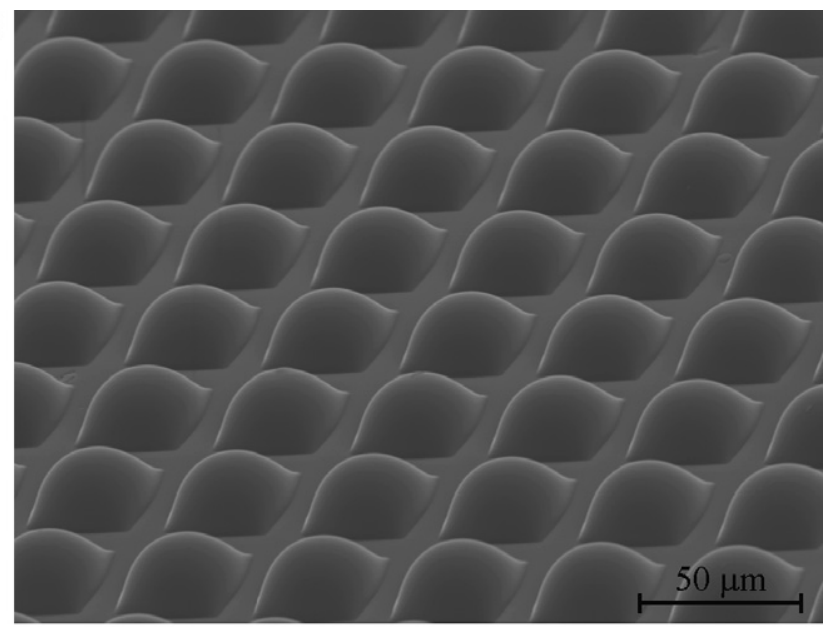

b

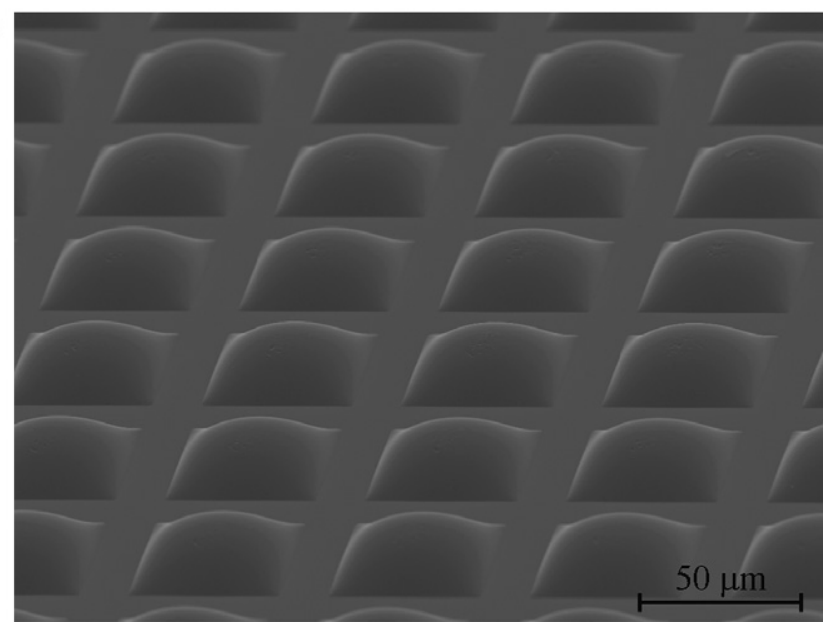

Fig. 3. Microphotographs of square-based microlens arrays, having a fill factor of $60.0 \%$, with different edge lengths: (a) $35.4 \mu \mathrm{m}$ and (b) $50.0 \mu \mathrm{m}$ (tilt angle: $\sim 60^{\circ}$ ).

the values of more than 10 different microlenses, the measured values were listed in Table 1 and Fig. 2. The microlens with a larger edge length possessed also a greater sag and radius of curvature, but a smaller height ratio. These trends were similar to those simulated from Eqs. (1a) and (1b).

When the OLED without any microlenses was driven at a luminance of $\sim 680 \mathrm{~cd} / \mathrm{m}^{2}$ at surface normal, the peak wavelength and the full width at half maximum (FWHM) of the spectrum were 515 and $95.6 \mathrm{~nm}$, respectively, and the CIE coordinate was $(0.2742,0.5317)[15]$. The luminance of the OLED decreased with increasing the viewing angle, as shown in Fig. 4, which is determined by the thin film organic layers, metal cathode, and the thick glass substrate $[14,15,18,19]$. Besides, the optical spectrum blue shifted and the FWHM narrowed as the viewing angle increased, as described in Fig. 5. The CIE- $x$ index decreased, and the CIE- $y$ index increased first and then decreased with increasing the fill factor, as illustrated in Fig. 6.

To illustrate the influences of the fill factor and the height ratio of microlenses on the efficiency improvement of the OLED, a ray tracing method (LightTools software) was used to simulate and qualitatively explain the propagation of light rays from an assumed Lambertian OLED device and that attached with microlenses. Due to the microcavity effect from OLED thin film structure, OLED is a non-Lambertian emitter actually. Hence, we can only obtain qualitative trends, rather than quantitative results. However, to model a non-Lambertian emitter is out of the scope of this paper. 


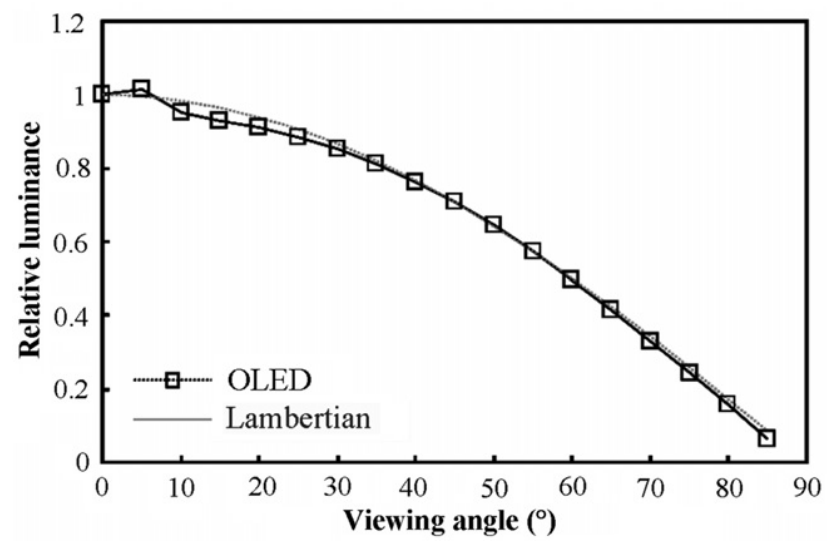

Fig. 4. The angular-dependent luminance of a green OLED without microlenses.
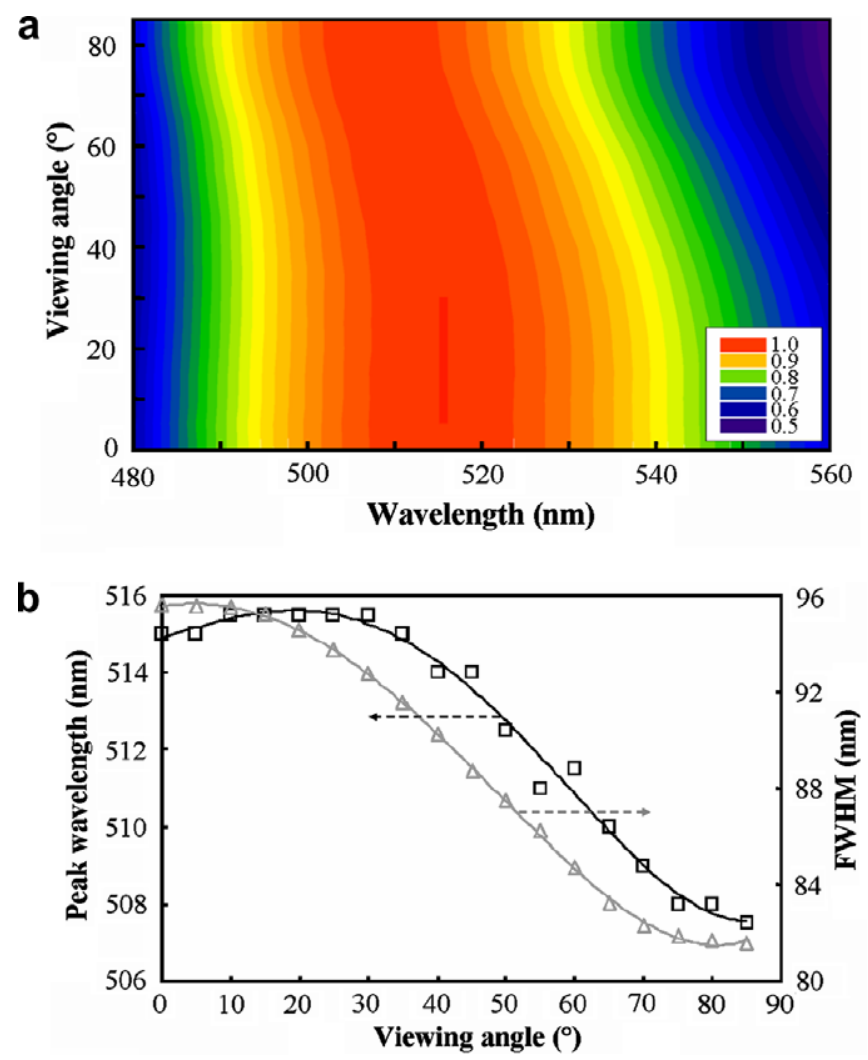

Fig. 5. (a) $2 \mathrm{D}$ plot of the OLED (without microlenses) intensity as a function of wavelength and viewing angle. (b) The variations of the peak wavelength and the FWHM of the OLED spectrum with the viewing angle.

The round-based spherical microlens arrays were used here instead of square-based ones due to lacking of exact contours of microlenses. Without microlenses attachment, the light rays emitted from the emitting layer of the OLED are reflected back at the glass/air interface when the incident angle is larger than the critical angle and then are reflected back to the front again by the cathode with assumed $100 \%$ reflection, as shown in Fig. 7a. As microlenses, having a diameter and a height ratio of $50 \mu \mathrm{m}$ and 1 , respectively, attached to the OLED, the light rays are possibly coupled out even at incident angles larger than the critical angle. As the fill factor of microlenses increases from $0 \%$ to $20 \%$ and then to $90 \%$, corresponding to Fig. $7 a-c$, respectively, the number of outcoupled light rays of the OLED increases accordingly. When the

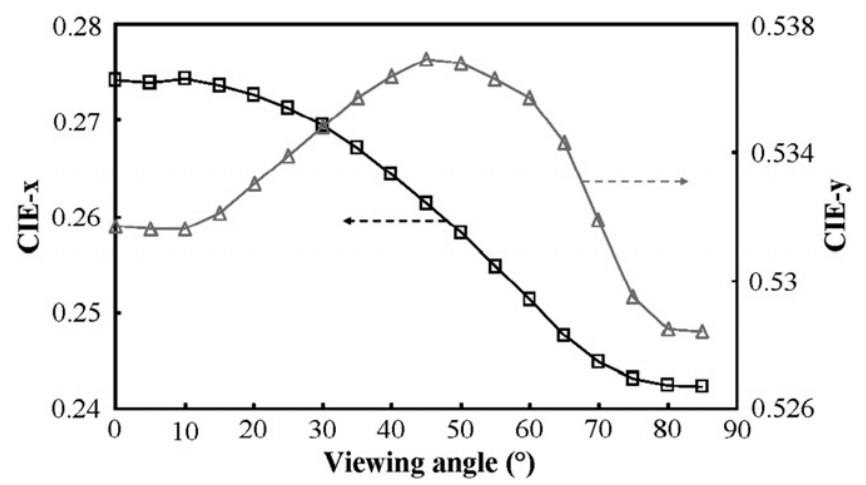

Fig. 6. The relationship between the CIE coordinate of the OLED without microlenses and the viewing angle.

OLED attached with microlenses having a diameter and a fill factor of $50 \mu \mathrm{m}$ and $90 \%$, respectively, we can find that as the height ratio of microlenses increases from 0 to 0.5 and then to 1 , referred to Fig. 7a, $d$ and c, respectively, the number of light rays of the OLED also increases. These simulated results are similar to those obtained by Greiner [14]. Of course, the reflectance of the cathode affects the amount of light rays which can be extracted into air. Fig. 8 is the simulation results by varying the reflectance of the cathode. The luminance normal to the emitting surface decreases as the cathode reflectance of the OLED is decreased.

As microlens arrays were attached in turn to the OLED, the luminance decreased with increasing the viewing angles, but increased with increasing the fill factor for both the two sets of microlenses, as shown in Fig. 9. These results confirmed our simulated results. In addition, the attached microlenses with a smaller edge length can increase more luminance of the OLED. These trends are similar to those in the previous work [13] which the hexagon-based microlens arrays were used. As illustrated before, microlenses with a larger edge length possessed also a larger sag but a smaller height ratio if the thicknesses of photoresist plates kept the same before thermal reflow. In this case, the sag itself can not explain the luminance variation of the OLED since the radius of curvature of attached microlenses also varies with the edge length. In fact, the height ratio, defined as the ratio of the lens sag to the radius of curvature, of microlenses is better than both the sag and the edge length to describe the luminance variation of the OLED, since a microlens with a larger height ratio has also a larger curved surface area. Therefore, the luminance of the OLED increased with increasing the height ratio of microlenses, which agreed to our simulated results.

Since the profile of the angular-dependent luminance of our OLED without microlenses is very similar to that of a Lambertian emitter, as depicted in Fig. 5a, the luminous current efficiency of the OLED can be determined from its luminance and driving current density. It also should be mentioned that the ratio of the luminous current efficiencies of the OLED with microlenses to that without microlenses is equal to the ratio of their luminance, as illustrated in Eq. (2). Therefore, the luminous current efficiency of the OLED with microlenses can be simply evaluated by dividing its luminance at perpendicular emission by that of the OLED without microlenses. Besides, the ratio of the luminous power efficiencies of the OLED with microlenses to that of the OLED without microlenses equals the ratio of their luminous exitances, as described in Eq. (3). The luminous exitance of the device can be obtained by integrating the product of its luminance and the sine function of the viewing angle at all directions. The luminous power efficiency of the OLED with microlenses can thus be expressed by the ratio of its luminous exitance to that of the OLED without microlenses. 
a

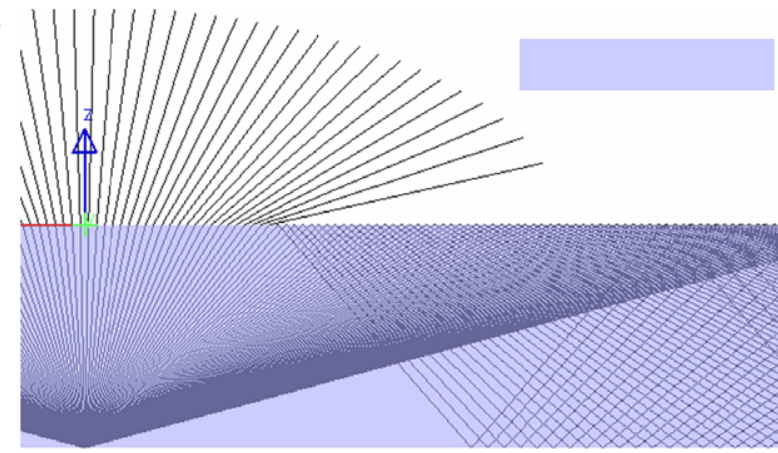

C

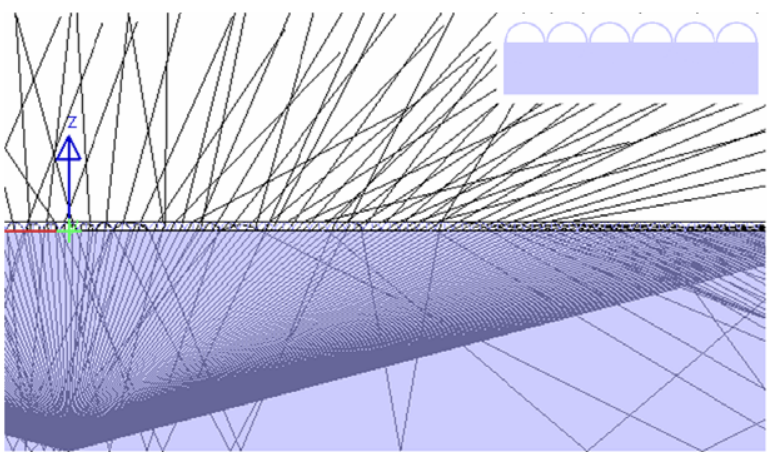

b

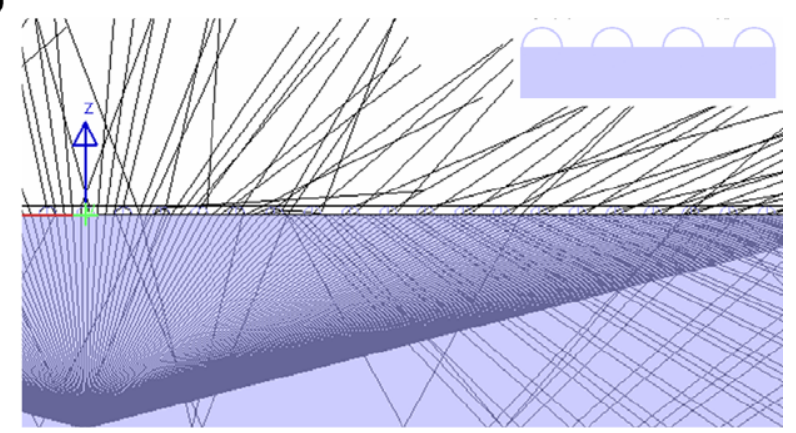

d

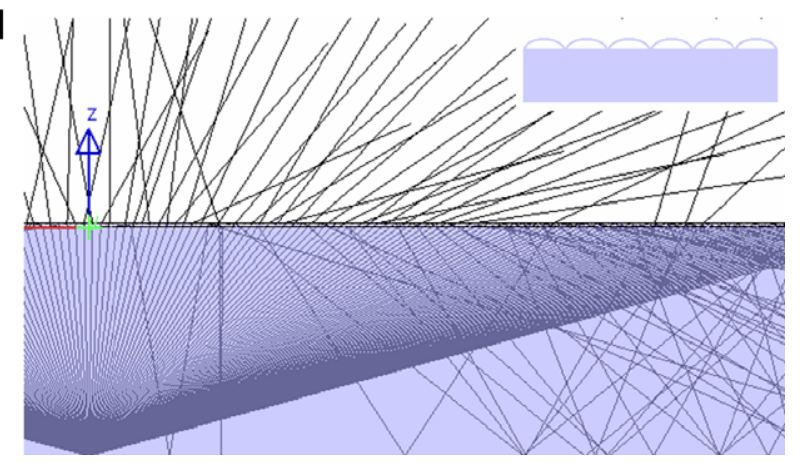

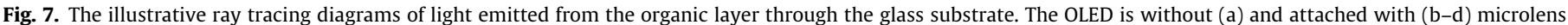
array. The diameter of microlenses is $50 \mu \mathrm{m}$. The fill factor and the height ratio of microlens arrays are (b) $20 \%$ and 1 , (c) $90 \%$ and 1 , and (d) $90 \%$ and 0.5 , respectively.

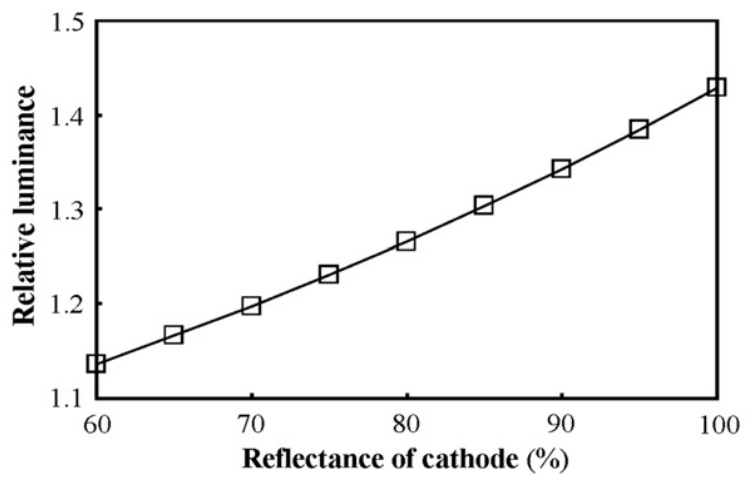

Fig. 8. The simulated relative luminance at surface normal versus the cathode reflectance of the OLED with microlens arrays. The base diameter, lens sag and gap distance were set at 50,17 and $3 \mu \mathrm{m}$, respectively.

Fig. 10 shows the behaviors of the luminous current efficiency (perpendicular to the OLED surface) and the luminous power efficiency versus the fill factor of attached microlenses. Both the luminous current efficiency and the luminous power efficiency increased with increasing not only the fill factor but also the height ratio. These trends are similar to those reported in our previous work [13] when using hexagon-based microlens arrays. The luminous current efficiency and the luminous power efficiency of the OLED can be increased up to $42 \%$ and $47 \%$, respectively, when the attached square-based microlenses had a fill factor and a height ratio of $90 \%$ and 0.57 , respectively. These values are greater than the reported values of $35 \%$ and $40 \%$ for the improvement of the luminous current efficiency and luminous power efficiency, respectively, of the OLED when attached with hexagon-based microlenses having a fill factor of 0.9 and a height ratio of 0.56 [13]. Though the square-based photoresist plates in this study had the same thickness as the hexagon-based ones (HO25) in the Ref. [13] before thermal reflow, the square-based microlenses did
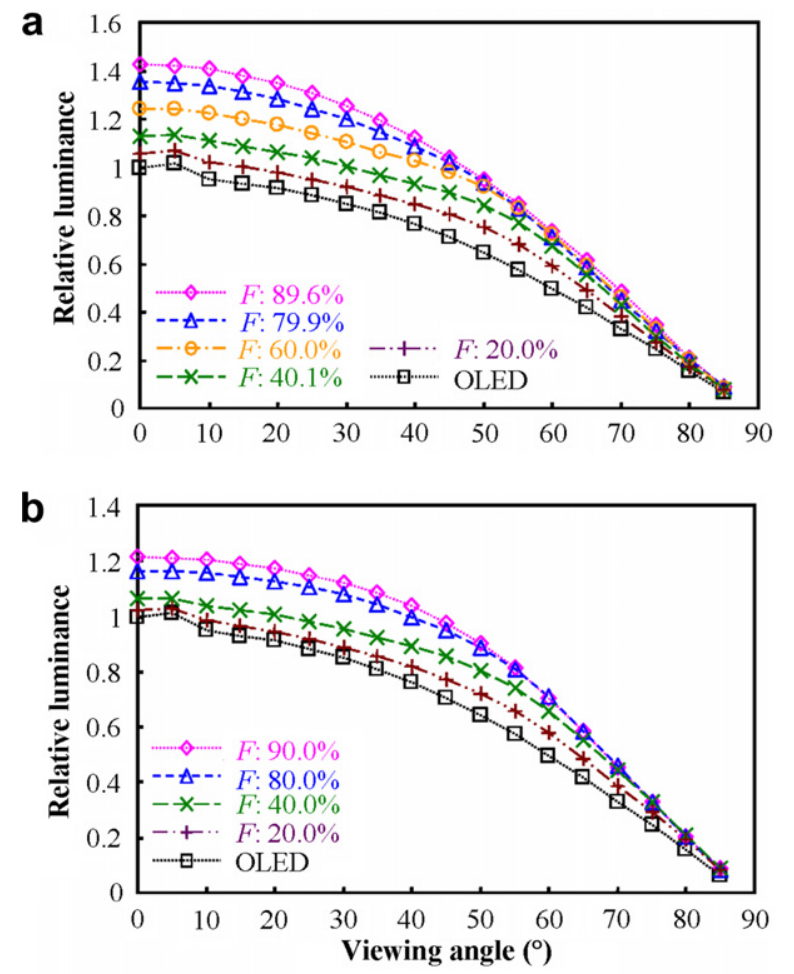

Fig. 9. The relative angular-dependent luminance of the OLED with microlens arrays having edge lengths of (a) 35.4 and (b) $50.0 \mu \mathrm{m}$, respectively.

have a greater height ratio than the hexagon-based ones after thermal reflow. Therefore, the square-based microlens arrays can enhance more efficiency of the OLED than the hexagon-based ones when having the same fill factor. 

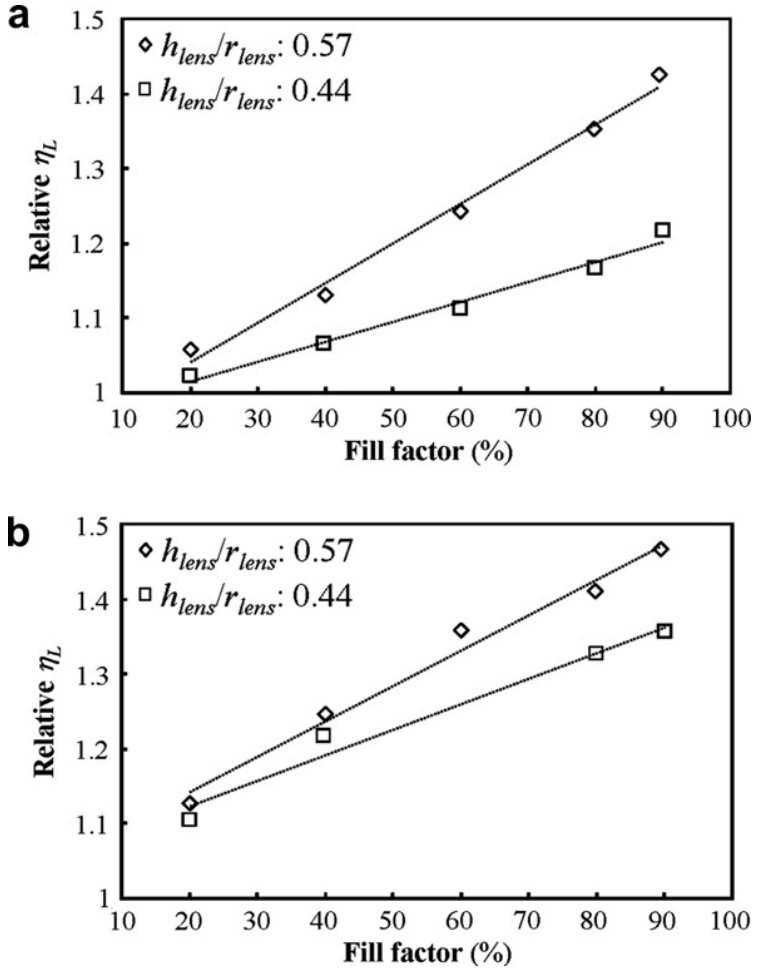

Fig. 10. The variations of (a) the luminous current efficiency and (b) the luminous power efficiency of the OLED with the attached microlenses having different height ratios.

It is important to note that the outcoupling efficiency enhancement depends not only on the microstructures attached on the device, but also on the thin film structures of the OLED itself, which determines the angular intensity and spectral distributions of light emission $[14,20]$. In this work, we fixed the OLED structure and varied the microlens parameters. Outcoupling efficiency enhancement shown here can be used to optimize the microlens design, but the value may be changed when the OLED structure is varied. However, as compared with our previous work (Ref. [15]) where we used the same OLED, we can see an obvious efficiency improvement when using microlens (42\%) rather than commercial diffuser BS-702 (29\%). Analyzing the data in Fig. 10, proportional relationships between the luminous current efficiencies $\left(\eta_{\mathrm{L}}\right)$, the luminous power efficiency $\left(\eta_{\mathrm{P}}\right)$ and the fill factors $(F)$ can be formulated

$\frac{\eta_{\mathrm{L}, \mathrm{lens}}}{\eta_{\mathrm{L}, 0}}=\frac{L_{\mathrm{lens}}}{L_{0}}=k_{1} F+k_{2}$

$\frac{\eta_{\mathrm{P}, \text { lens }}}{\eta_{\mathrm{P}, 0}}=k_{3} F+k_{4}$

where $k_{1}$ and $k_{3}$ represent the slopes, and $k_{2}$ and $k_{4}$ are the constants in these curves, as listed in Table 2. As the fill factor approaches zero, there are no microlenses on the duplicated films. Thus, $k_{2}$ and $k_{4}$ should approach unity. In fact, the values of $k_{2}$ and $k_{4}$ in our results were close to unity. If both $k_{2}$ and $k_{4}$ equal unity, Eqs. (5a) and (5b) can then be rewritten as

Table 2

The values of $k_{1}, k_{2}, k_{3}$ and $k_{4}$

\begin{tabular}{lllll}
\hline Set no. & $k_{1}$ & $k_{2}$ & $k_{3}$ & $k_{4}$ \\
\hline RO35.4 & 0.530 & 0.935 & 0.472 & 1.048 \\
RI50 & 0.266 & 0.962 & 0.342 & 1.054 \\
\hline
\end{tabular}

$$
\begin{aligned}
& \frac{\eta_{\mathrm{L}, \text { lens }}-\eta_{\mathrm{L}, 0}}{\eta_{\mathrm{L}, 0}}=k_{1} F \\
& \frac{\eta_{\mathrm{P}, \text { lens }}-\eta_{\mathrm{P}, 0}}{\eta_{\mathrm{P}, 0}}=k_{3} F
\end{aligned}
$$

Thus, $k_{1}$ and $k_{3}$ are the enhancement factors of the luminous current efficiency and the luminous power efficiency of the OLED, respectively, when the emitting surface of the device is fully occupied with microlenses. As illustrated in Table 2, both the enhancement factors of the luminous current efficiency and the luminous power efficiency of the OLED increased with increasing the height ratio of attached microlenses. These trends are also similar to those reported in the Ref. [13] when using the hexagon-based microlens arrays. Besides, these trends remain true regardless of the base shapes of microlenses.

The angular-dependent spectra of the OLED with microlens arrays, having the same fill factor of $90 \%$ and different sags, were shown in Fig. 11. Blue shifts, compared to the spectrum of the OLED without microlenses, were observed at the normal direction. The peak wavelengths shifted $-3.0 \mathrm{~nm}$ and $-1.0 \mathrm{~nm}$, and the FWHMs were narrowed by $-4.5 \mathrm{~nm}$ and $-2.8 \mathrm{~nm}$ when the attached microlens arrays had height ratios of 0.57 and 0.44 , respectively. Besides, the spectral shift of the OLED increased with increasing both the fill factor and the height ratio of microlenses.

There are several possible mechanisms for the angular-dependent spectral shift when attaching the microlens array film, such as the material dispersion of glass and organic thin films and hence wavelength dependent angle of total reflection. Since the refractive indices are a decreasing function of the wavelength, the light cone to be extracted from the surface of the substrate will be an increasing function of the wavelength. From this viewpoint, there will be red shift for increasing viewing angle. The other mechanism is the intrinsic angular characteristics of the OLED itself [20]. The observed variability of the spectral data can be explained in the
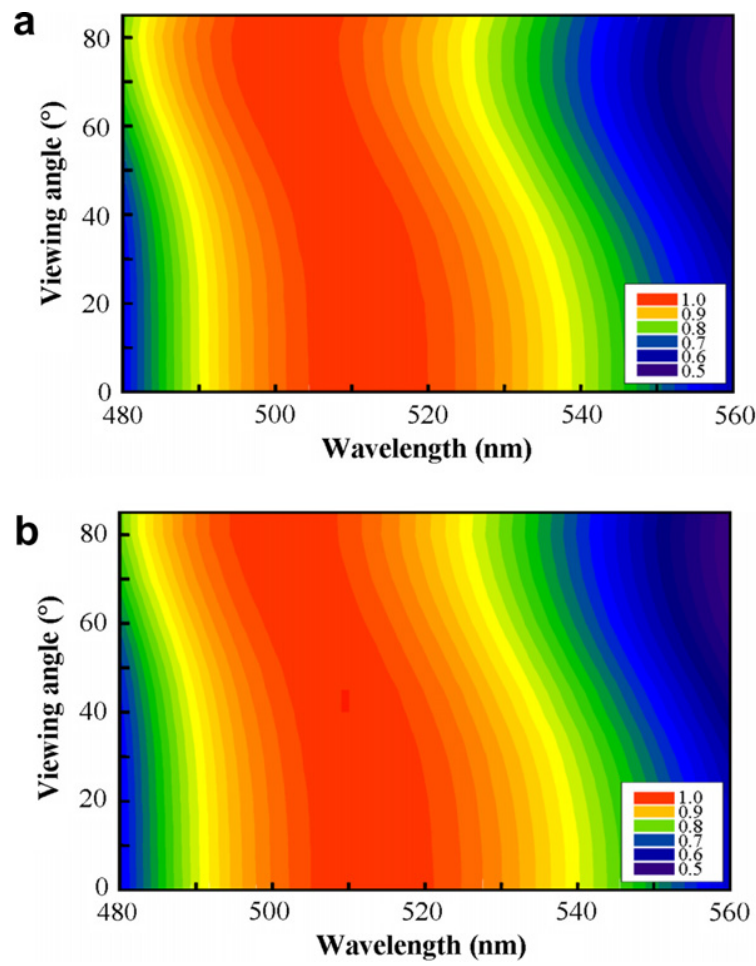

Fig. 11. 2D plot of the spectral intensity of the OLED with microlenses as a function of wavelength and viewing angle. The microlenses had a fill factor of $90 \%$, and their height ratios were (a) 0.57 and (b) 0.44 , respectively. 
framework of the general model, but is too weak to be attributed to specific causes. More detailed simulations would be required which are however out of the scope of this paper. Besides, we did observe blue or red shift behaviors when attaching microlens arrays on OLED depending on their layer structures, i.e. angular characteristics, not shown here. As shown in [20], for certain thin film structure, the waveguided light contains more "blue" components. When the OLED is attached with microlenses, the waveguiding effect is decreased and the light in the substrate mode is coupled out.

Apart from spectral shift, the FWHM of the OLED decreased with increasing both the fill factor and the height ratio of attached microlenses, as illustrated in Fig. 12. That is, the emitting color of the OLED gets purer when microlens arrays are arranged more densely and have a larger height ratio. Besides, the FWHM of the device decreased with increasing the viewing angle. For the viewing angle less than $60^{\circ}$, proportional relationships between the FWHMs and the fill factors can be found as below.

$\mathrm{FWHM}=k_{5} F+k_{6}$

where $k_{5}$ and $k_{6}$ represent the slope and constant in these curves, respectively. All the values of $k_{5}$ and $k_{6}$ at different viewing angles were listed in Tables 3 and 4 . If the device is attached without any microlenses, the value of $k_{6}$ should approach the FWHM of the OLED. In our results, the values of $k_{6}$ were very close to the FWHM of the OLED. Thus, $k_{5}$ is the difference of the FWHMs between the OLED without and fully covered with microlenses. As listed in Tables 3 and 4, the larger the height ratio of microlenses, the smaller the value of $k_{5}$.

The behavior of the CIE coordinates of the OLED versus the fill factors of attached microlenses at different viewing angles were shown in Figs. 13 and 14. All the CIE- $x$ and CIE- $y$ indices of the OLED decreased with increasing both the fill factor and the height ratio of attached microlenses. Analyzing the data in Figs. 13 and 14,
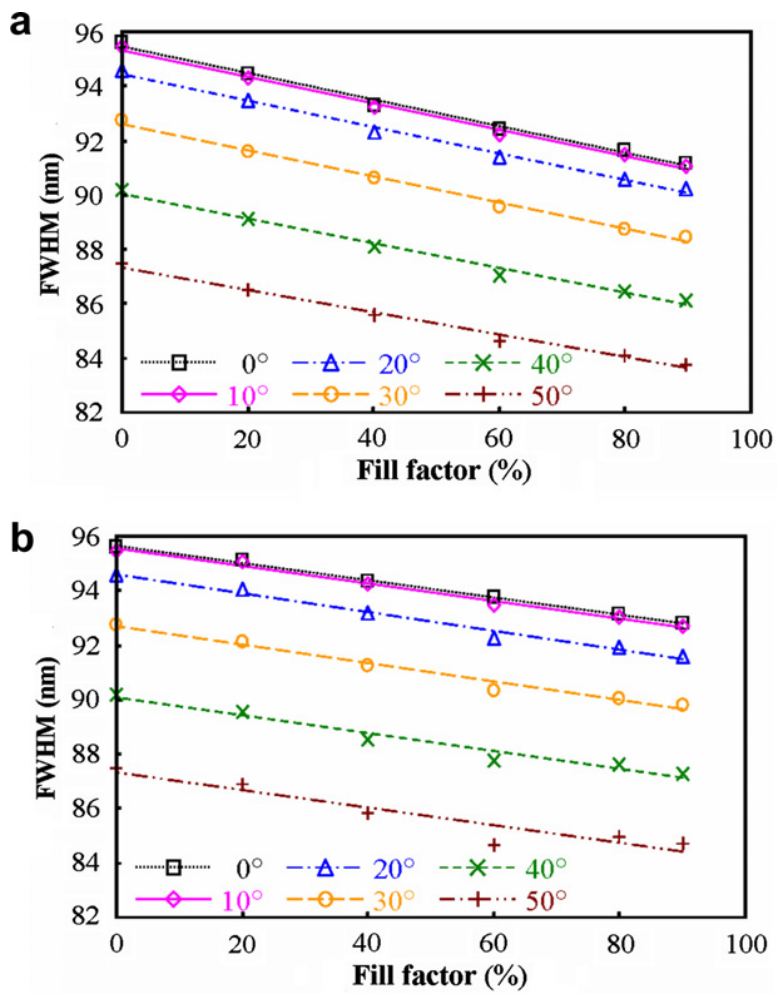

Fig. 12. The variation of the FWHM of the OLED spectra with the fill factor of microlenses having height ratios of (a) 0.57 and (b) 0.44 , respectively.
Table 3

The values of $k_{5}, k_{6}, k_{7}, k_{8}, k_{9}$ and $k_{10}$ for the OLED attached with microlenses (RO35.4)

\begin{tabular}{llllllll}
\hline Set no. & $\begin{array}{l}\text { Viewing } \\
\text { angle }\left(^{\circ}\right)\end{array}$ & $k_{5}$ & $k_{6}$ & $k_{7}$ & $k_{8}$ & $k_{9}$ & $k_{10}$ \\
\hline RO35.4 & 0 & -4.903 & 95.46 & -0.0121 & 0.2743 & -0.0004 & 0.5324 \\
& 10 & -4.880 & 95.32 & -0.0125 & 0.2743 & -0.0004 & 0.5323 \\
& 20 & -4.852 & 94.44 & -0.0130 & 0.2728 & -0.0018 & 0.5336 \\
& 30 & -4.826 & 92.62 & -0.0141 & 0.2695 & -0.0044 & 0.5353 \\
& 40 & -4.570 & 90.05 & -0.0148 & 0.2639 & -0.0089 & 0.5367 \\
& 50 & -4.145 & 87.34 & -0.0155 & 0.2573 & -0.0158 & 0.5365 \\
\hline
\end{tabular}

Table 4

The values of $k_{5}, k_{6}, k_{7}, k_{8}, k_{9}$ and $k_{10}$ for the OLED attached with microlenses (RI50)

\begin{tabular}{llllllll}
\hline $\begin{array}{l}\text { Set } \\
\text { no. }\end{array}$ & $\begin{array}{l}\text { Viewing angle } \\
\left({ }^{\circ}\right)\end{array}$ & $k_{5}$ & $k_{6}$ & $k_{7}$ & $k_{8}$ & $k_{9}$ & $k_{10}$ \\
\hline RI50 & 0 & -3.157 & 95.66 & -0.0083 & 0.2747 & -0.0006 & 0.5321 \\
& 10 & -3.195 & 95.55 & -0.0089 & 0.2747 & -0.0007 & 0.5320 \\
& 20 & -3.423 & 94.59 & -0.0099 & 0.2730 & -0.0020 & 0.5333 \\
& 30 & -3.416 & 92.71 & -0.0110 & 0.2696 & -0.0042 & 0.5351 \\
& 40 & -3.299 & 90.09 & -0.0117 & 0.2639 & -0.0075 & 0.5365 \\
& 50 & -3.236 & 87.32 & -0.0123 & 0.2574 & -0.0121 & 0.5363 \\
\hline
\end{tabular}

a
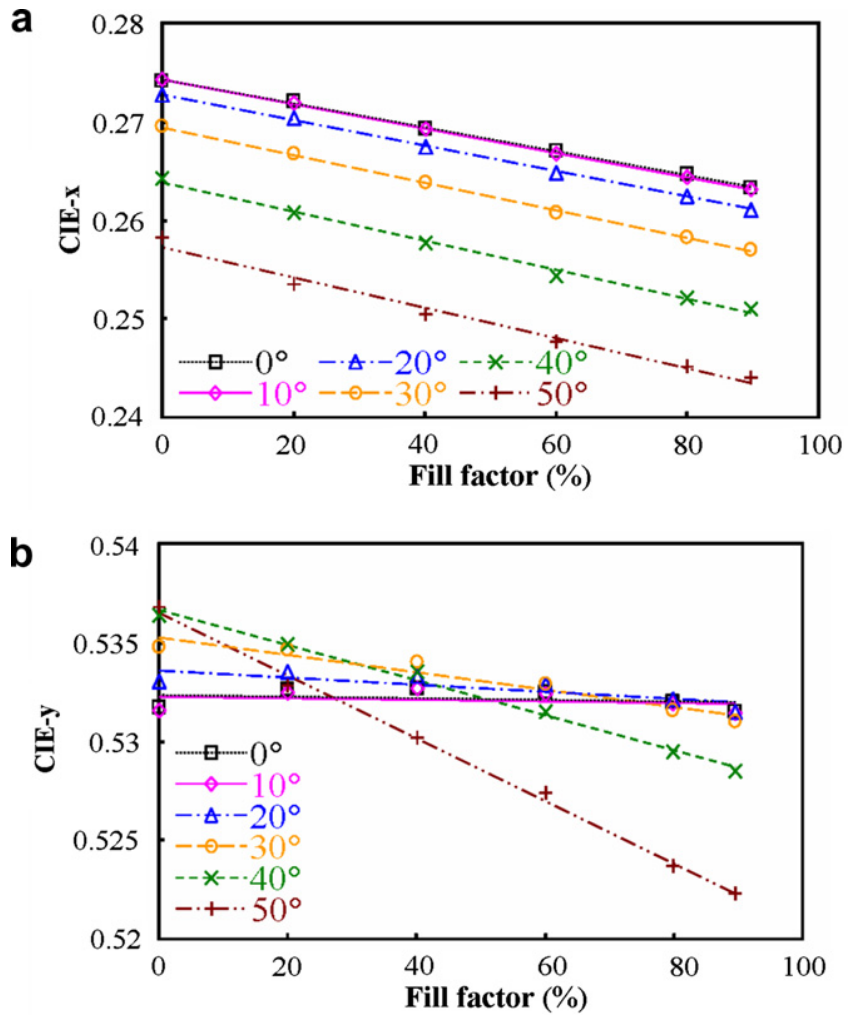

Fig. 13. The behaviors of (a) the CIE- $x$ and (b) the CIE- $y$ indices of the OLED versus the fill factor of microlenses having a height ratio of 0.57 .

proportional relationships between the CIE coordinates and the fill factors can be formulated

$\mathrm{CIE}-x=k_{7} F+k_{8}$

$\mathrm{CIE}-y=k_{9} F+k_{10}$

where $k_{7}$ and $k_{9}$, and $k_{8}$ and $k_{10}$ represent the slopes and constants in these curves of the CIE- $x$ and CIE- $y$ indices versus the fill factor, respectively. All the values of $k_{7}, k_{8}, k_{9}$ and $k_{10}$ at different viewing 

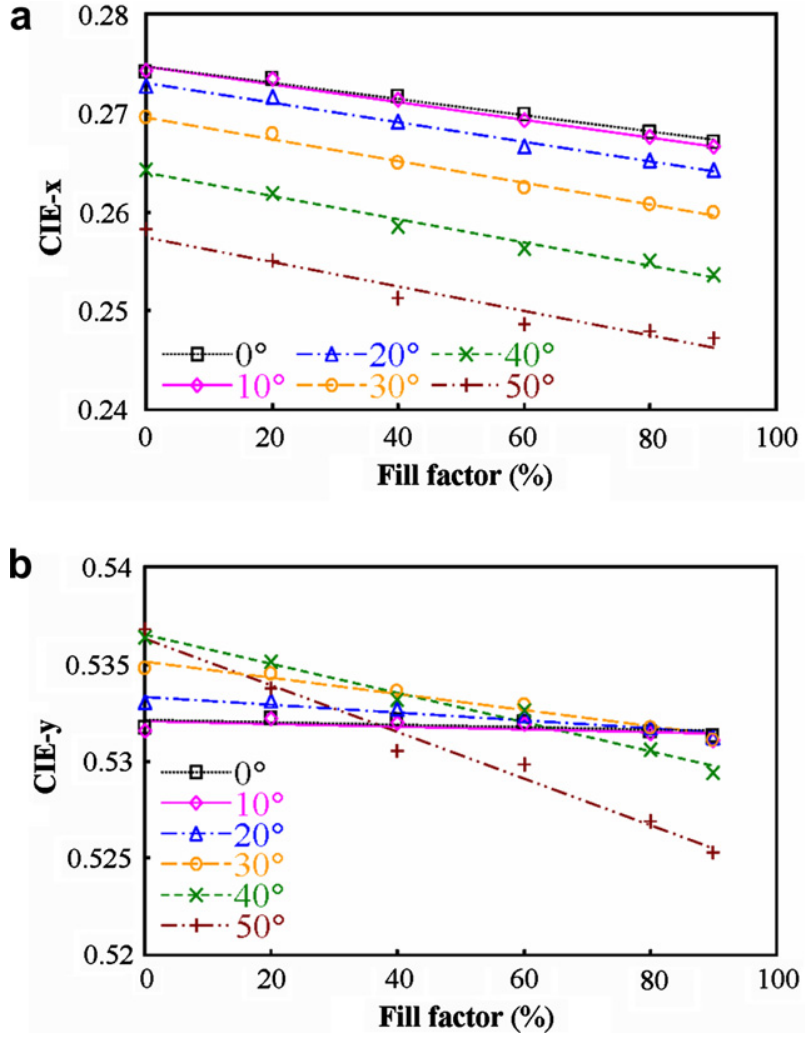

Fig. 14. The behaviors of (a) the CIE- $x$ and (b) the CIE- $y$ indices of the OLED versus the fill factor of microlenses having a height ratio of 0.44 .

angles were listed in Tables 3 and 4 . As the fill factor approaches zero, $k_{8}$ and $k_{10}$ should approach the CIE- $x$ and CIE- $y$ indices of the OLED without microlenses, respectively. It agreed to our results. Thus, $k_{7}$ and $k_{9}$ are the maximal shifts of the CIE- $x$ and CIE- $y$ indices of the OLED when the device is fully covered with microlenses. As listed in Tables 3 and $4, k_{7}$ and $k_{9}$ decreased with increasing the height ratio of microlenses.

As illustrate in the Ref. [15], the diffuser film BS-702 can enhance the luminous current efficiency and luminous power efficiency of the OLED by $29 \%$ and $28 \%$, respectively. Compared to the BS-702, our square-based microlens array, having a height ratio and a fill factor of 0.57 and $90 \%$ respectively, can improve much more efficiency of the OLED. Additionally, the spectral shift and the FWHM narrowing of the OLED attached with this microlens array were also smaller. Therefore, square-based microlens arrays seem to be better than the BS-702 in the backlight applications of the OLEDs.

\section{Conclusions}

Square-based microlens arrays with various fill factors and sags have been made. They were used to improve both the luminous current efficiency and luminous power efficiency of a planar OLED in purpose. Results showed that the luminous current efficiency and luminous power efficiency of the OLED have been enhanced by $42 \%$ and $47 \%$, respectively. It can be expected to further increase the efficiency of the OLED by increasing the height ratio of attached microlenses. Results also showed that both the luminous current efficiency and luminous power efficiency of the OLED increased linearly with increasing the fill factor of microlenses. But all the FWHMs of the spectra and the CIE coordinates of the device decreased linearly with increasing the fill factor of microlenses. In summary, high fill factor and large height ratio of microlens arrays are expected to increase the efficiency of the OLED.

\section{Acknowledgments}

The authors gratefully acknowledge the financial support given by the National Science Council of the Republic of China under the Project No. NSC 97-2221-E-259-009, NSC 96-2221-E-002-118 and NSC 97-2221-E-002-056. We also acknowledge the financial support given by the Chung-Shan Institute of Science and Technology of the Republic of China.

\section{References}

[1] M.-H. Lu, J.C. Sturm, Appl. Phys. Lett. 78 (2001) 1927.

[2] G. Gu, D.Z. Garbuzov, P.E. Burrows, S. Venkatesh, S.R. Forrest, Opt. Lett. 22 (1997) 396.

[3] T. Yamasaki, K. Sumioka, T. Tsutsui, Appl. Phys. Lett. 76 (2000) 1243

[4] L. Lin, T.K. Shia, C.-J. Chiu, J. Micromech. Microeng. 10 (2000) 395.

[5] T. Tsutsui, M. Yahiro, H. Yokogawa, K. Kawano, M. Yokoyamma, Adv. Mater. 13 (2001) 1149

[6] J.J. Shiang, T.J. Faircloth, A.R. Duggal, J. Appl. Phys. 95 (2004) 2889.

[7] C.F. Madigan, M.-H. Lu, J.C. Sturm, Appl. Phys. Lett. 76 (2000) 1650

[8] S. Möller, S.R. Forrest, J. Appl. Phys. 91 (2002) 3324.

[9] M.-K. Wei, I-L. Su, Opt. Express 12 (2004) 5777.

[10] H. Peng, Y.L. Ho, X.-J. Yu, M. Wong, H.-S. Kwok, J. Display Technol. 1 (2005) 278.

[11] Y. Sun, S.R. Forrest, J. Appl. Phys. 100 (2006) 073106.

[12] M.-K. Wei, I-L. Su, Y.-J. Chen, M. Chang, H.-Y. Lin, T.-C. Wu, J. Micromech. Microeng. 16 (2006) 368.

[13] M.-K. Wei, J.-H. Lee, H.-Y. Lin, Y.-H. Ho, K.-Y. Chen, C.-C. Lin, C.-F. Wu, H.-Y. Lin, J.-H. Tsai, T.-C. Wu, J. Opt. A: Pure Appl. Opt. 10 (2008) 055302.

[14] H. Greiner, Jpn. J. Appl. Phys. 46 (2007) 4125.

[15] H.-Y. Lin, J.-H. Lee, M.-K. Wei, C.-L. Dai, C.-F. Wu, Y.-H. Ho, H.-Y. Lin, T.-C. Wu, Opt. Commun. 275 (2007) 464.

[16] F.T. O’Neill, J.T. Sheridan, Optik 113 (2002) 391.

[17] M. He, X.-C. Yuan, N.Q. Ngo, W.C. Cheong, J. Bu, Opt. Eng. 42 (2003) 2180.

[18] J.-H. Lee, X. Zhu, Y.-H. Lin, W.-K. Choi, T.-C. Lin, S.-C. Hsu, H.-Y. Lin, S.-T. Wu, Opt. Express 13 (2005) 9431.

[19] H.-C. Chen, J.-H. Lee, C.-C. Hsiao, C.C. Yang, Y.-W. Kiang, J. Lightwave Technol. 24 (2006) 2450.

[20] B.C. Krummacher, M.K. Mathai, V. Choong, S.A. Choulis, F. So, A. Winnacker, J. Appl. Phys. 100 (2006) 054702. 